\title{
A formal model for the discrete representation of spatial objects*
}

\author{
Francesca Coppa, ${ }^{1,3}$ Enrico Nardelli, ${ }^{1,2}$ and Maurizio Talamo ${ }^{3}$
}

1. Dipartimento di Matematica Pura ed Applicata, Università di L'Aquila, Via Vetoio, Coppito, I-67010

L'Aquila, Italia, e-mail: nardelli@univaq.it.

2. Istituto di Analisi dei Sistemi ed Informatica, C.N.R., Viale Manzoni 30, 00185 Roma, Italia.

3. Dipartimento di Informatica e Sistemistica, Università di Roma "La Sapienza", Via Salaria 113, I-00197

Roma, Italia, e-mail: talamo@ disparcs.dis.uniromal.it.

Keywords: spatial databases, data models, spatial relations.

\begin{abstract}
In this paper we define a formal model for the discrete representation of spatial objects and characterize its properties. The model and its manipulation primitives are based only on set theory and do not use any metricbased concept. A general characterization for containment and intersection relations is given. The model is based on a mapping from spatial objects to their representations as sets of points of $Z^{2}$ such that queries on spatial objects can be answered by applying simple set-theoretic primitives to their corresponding discrete representations. The mapping makes reference to a suitable topology whose underlying set is a canonical decomposition of the real plane in square cells. But the introduced model keep its properties under every homeomorphic topological space such that its underlying set is a countable partition of the plane whose cells are simply connected.
\end{abstract}

\section{INTRODUCTION}

The definition of a formal framework for the representation and manipulation of spatial objects and

(*) Work partially supported by the italian MURST $40 \%$ Project "Algoritmi, Modelli di Calcolo e Strutture Informative", by the italian CNR Coordinated Project "Modelli e Sistemi per il Trattamento di Dati Ambientali e Territoriali", and by the European Union's Research Network CHOROCHRONOS on SpatioTemporal Dalabases.

"Permission to make digital or hard copies of part or all of this work for personal or classroom use is granted without fee provided that copies are not made or distributed for profit or commercial advantage and that copies bear this notice and the full citation on the tirst page. Copyrights for components of this work owned by others than ACM must be honored. Abstracting with credit is permitted. To copy otherwise to republish. to post on servers or to redistribute to lists, requires prior specitic permission and/or a tee."

$c .1997$ ACM 0-89791-850-9 $97 \quad 0002 \quad 3.50$ their relations is a key issue in the theory of spatial databases. Since we want to use computing devices to represent and manipulate spatial objects and their relations it is of the greatest importance to focus on finite formalizations.

In this paper we are therefore concerned with the definition of a theory which models spatial relations among geographical object and is, at the same time, based on finite and discrete representations of spatial objects.

Many different models for spatial objects and relations have been introduced in the literature. Among the most widely known there is the Egenhofer and Franzosa's 4intersection model [EF91] and its extensions ([Her91], [CDO93], [ES93]), all based on point-set topology.

Other interesting models formalizing spatial relations are based on simplicial complexes ([EFJ89], [Wor92]) or on partial orders or on a combination of them [KEG].

Considering finite representation of spatial objects, Guibas, Salesin and Stolfi defined Epsilon-Geometry [GSS89, GSS93], which allows to carry out exact geometric computations even when only inaccurate primitives are available. This approach, while very good in dealing with building accurate discrete representation of real objects does not explicitly address issues of spatial relations. Greene and Yao [GY86] introduced a computational geometry framework for the topologically correct computation of intersection (discrete) points in a set of line segments whose endpoints have discrete coordinates. A completely different approach was taken by Güting and Schneider [GS93]. They introduced the concept of Realm (i.e., a set of points and non-intersecting lines over a discrete domain)which allows to model spatial relations through an algebraic approach. Discrete representations at the Realm level are derived from real objects by 
means of computational geometry primitives which take into account rounding problems. Representations at the Realm level are then manipulated through a very clean algebraic interface, where spatial relations are defined by means of a algebraic specifications. But the introduction of an intermediate level, while allowing a very good formalization of spatial relations and manipulations, hides the true relations between objects in the real world and their counterparts in the model.

In this paper we address these issues by defining a formal model for spatial objects and relations and characterizing its properties. We have already introduced in [CNT96] a discrete representation for spatial relations which allows to test containment and intersection relations among convex polygons. We defined a topology preserving mapping from the set of convex polygons to a discrete space. Here we extend and refine that approach. Objects we consider are arbitrary polygons and lines whose vertices are points with discrete-valued coordinates.

The discrete space we use as basis for our framework is a countable set of points whose coordinates are suitably taken from the set of rational numbers. We define a correspondence between this discrete space and a partition of the continuous real plane in squares. This specific partition is chosen only for ease of formalization, since our results hold for any decomposition of the real plane that is a partition in cells such that the cells are the underlying set of a topological space homeomorphic to the one we introduce.

The manipulation primitives introduced in the model are purely topological and based on set-theory, without using any concept based on metrics. Thus we are able to give a finite characterization of each of the three basic relative positions of spatial objects (namely: containment, intersection, and disjointness). These are the three fundamental relations to be managed by any efficient organization of spatial data.

The paper is structured as it follows. In section 2 we introduce the topological space used for the discrete framework and the mapping between it and real objects. In section 3 we characterize the containment relation between polygons. Section 4 completes the characterization by studying the intersection relation. Section 5 address generalization issues and, finally, section 6 contains conclusive remarks.

\section{BASIC DEFINITIONS}

In this section we introduce the topological space used for the discrete representation of objects and the one-toone mapping between it and objects in the reality. Let $\mathbf{N}, \mathbf{Z}, \mathbf{Q}, \mathbf{R}$ denote the sets of, respectively, natural, integer, rational, and real numbers. Each set is taken with its usual total order.

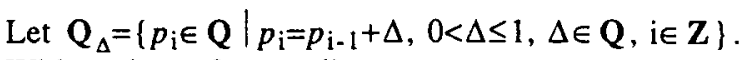
Without loss of generality, we can assume $p_{0}=0$. We note that on $\mathbf{Q}_{\Delta}$ a successor function succ: $\mathbf{Q}_{\Delta} \rightarrow \mathbf{Q}_{\Delta}$ can be defined. Given a $p_{\mathrm{i}} \in \mathbf{Q}_{\Delta}$ we let $\operatorname{succ}\left(p_{\mathrm{i}}\right)=p_{\mathrm{i}+1}$. Let ] $a, b$ [, with $a<b$, denote an open interval in $\mathbf{Q}_{\Delta}$, that is ]$a, b\left[=\left\{p \mid a<p<b, a, b, p \in \mathbf{Q}_{\Delta}\right\}\right.$.

Let $\mathcal{G}_{\Delta}=\mathbf{Q}_{\Delta} \times \mathbf{Q}_{\Delta}$ and let $\left(\mathcal{G}_{\Delta}, \mathcal{T}_{\Delta}\right)$ be the topological space whose open sets are generated by the following basis $\mathcal{B}_{\Delta}=\{] a, b[\times] c, d\left[\mid a, b, c, d \in \mathbf{Q}_{\Delta}\right\}$.

Let $T \in \mathcal{T}_{\Delta}$. We denote with $C(T)$ the complement in $\mathcal{G}_{\Delta}$ of $T$, i.e. the set $C(T)=\mathcal{G}_{\Delta} \backslash T$. We denote with $\bar{T}$ the closure of $T$, i.e. the intersection of all closed sets ${ }^{1}$ of $\left(G_{\Delta}, \mathcal{T}_{\Delta}\right)$ containing $T$. We denote with $\partial T$ the boundary of $T$, i.e. the set $\partial T=\bar{T} \cap \overline{C(T)}$.

DEFINITION 1: Let $T \in \mathcal{T}_{\Delta_{i}} T$ is called canonical if $T=\{] a_{\mathrm{i}}, a_{\mathrm{i}+1}[\times] b_{\mathrm{j}}, b_{\mathrm{j}+1}\left\{\mid\left(a_{\mathrm{i}}, b_{\mathrm{j}}\right) \in G_{\Delta}\right\} . T$ is called degenerate if $\{p \mid p \in T\}=\varnothing$.

Note that a canonical open set is always degenerate.

DEFINITION 2: Let $\mathbf{x} \in \mathcal{G}_{\Delta}$. We denote with $\mathcal{U}_{\mathbf{x}}$ the family of open sets of $\mathcal{T}_{\Delta}$ containing $x$. We call $U_{\mathbf{x}}=\bigcap_{v \in \mathcal{U}_{\mathrm{x}}} V$ the pointed neighbourhood of $\mathbf{x}$. $\odot$ DEFINITION 3: Let $\mathbf{x}=\left(x_{1}, x_{2}\right)$ and $\mathbf{y}=\left(y_{1}, y_{2}\right)$ belong to $\mathcal{G}_{\Delta}$. We say $\mathbf{x}$ is adjacent to $\mathbf{y}$ if $\left(x_{1}=\operatorname{succ}\left(y_{1}\right) \vee\right.$ $\left.y_{1}=\operatorname{succ}\left(x_{1}\right)\right) \wedge\left(x_{2}=y_{2}\right)$ or if $\left(x_{2}=\operatorname{succ}\left(y_{2}\right) \vee\right.$ $\left.y_{2}=\operatorname{succ}\left(x_{2}\right)\right) \wedge\left(x_{1}=y_{1}\right)$.

DEFINITION 4: Let $\gamma=\left\{\mathbf{x}_{1}, \mathbf{x}_{2}, \ldots, \mathbf{x}_{\mathrm{k}}\right\}, k \geq 2$, be a finite set of points of $G_{\Delta}$, such that $x_{i-1}$ and $x_{i}$ are adjacent for $i=2,3, \ldots, k$, and $\mathbf{x}_{\mathrm{i}} \neq \mathbf{x}_{\mathrm{j}}$ for $i \neq j$. We say $\gamma$ is a chain.

DEFINITION 5: Let $\mathbf{A}$ be a set of points of $\mathcal{G}_{\Delta}$. We say $A$ is connected if for each couple of distinct points $\mathbf{x}$ and $\mathbf{y}$ belonging to $A$ a chain $\gamma=\left\{x_{1}, x_{2}, \ldots, x_{k}\right\}$ exists such that $\mathbf{x}_{1}=\mathbf{x}$ and $\mathbf{x}_{\mathrm{k}}=\mathbf{y}$.

()

DEFINITION 6: Let $\lambda=\left\{\mathbf{x}_{1}, \mathbf{x}_{2}, \ldots, \mathbf{x}_{\mathrm{k}}\right\}, k \geq 4$, be a chain such that $\mathbf{x}_{1}=\mathbf{x}_{k}$. We say $\lambda$ is a loop. The (possibly

\footnotetext{
${ }^{1}$ A closed set of a topological space $(X, T)$ is a subset of $X$ such that its complement in $X$ is an open set of $(X, T)$.
} 
empty) union of all maximal connected finite subsets of the set $G_{\Delta} \backslash \lambda$ is denoted $\varphi(\lambda)$.

()

DEFINITION 7: Let $\mathrm{A}$ be a subset of the topological space $\left(\mathcal{G}_{\Delta}, \mathcal{T}_{\Delta}\right)$. A is called loop-connected if a loop $\lambda \subseteq A$ exists such that $\forall \mathbf{x} \in A$ it is $\mathbf{x} \in \lambda \cup \varphi(\lambda)$.

We can now consider the countable partition in disjoint sets induced on $\mathbf{R}^{2}$ by $\mathcal{G}_{\Delta}$. Such a partition is denoted $\mathbf{R}_{\Delta}^{2}$ in the following.

DEFINITION 8: Let $q=\left\{(x, y) \mid(x, y) \in \mathbf{R}^{2}, a_{\mathrm{i}} \leq x<a_{\mathrm{i}+1}\right.$, $\left.b_{\mathrm{j}} \leq y<b_{\mathrm{j}+1},\left(a_{\mathrm{i}}, b_{\mathrm{j}}\right) \in \mathcal{G}_{\Delta}\right\} \in \mathbf{R}_{\mathrm{u}}^{2}$. We say $\left(a_{\mathrm{i}}, b_{\mathrm{j}}\right)$ is the base point of $q$, and we let $h b p(q)=a_{\mathrm{i}}$ and $v b p(q)=b_{\mathrm{j}}$.

웅

It is naturally defined a one-to-one function $b p$ : $\mathbf{R}_{\Delta}^{2} \rightarrow \mathcal{G}_{\Delta}$ that associates at each $q$ of $\mathbf{R}_{\Delta}^{2}$ its base point $b p(q)$. We assume $\mathcal{G}_{\Delta}$ is provided with the topology $\mathcal{T}_{\Delta}$ above defined and we provide $\mathbf{R}_{\Delta}^{2}$ with the topology $\mathcal{T}_{\Delta}^{\prime}$ defined as the inverse image of $\mathcal{T}_{\Delta}$ under $b p$. To obtain $\mathcal{T}_{\Delta}^{\prime}$ we can consider its basis $\mathcal{B}_{\Delta}^{\prime}$ obtained as the inverse image of $\mathcal{B}_{\Delta}$ under $b p$. An element of $\mathcal{B}_{\Delta}^{\prime}$ is therefore an open set $A^{\prime}=\left\{q \in \mathbf{R}_{\Delta}^{2} \mid a \leq h b p(q)<b \wedge\right.$ $\left.c \leq v b p(q)<d, a, b, c, d \in \mathbf{Q}_{\Delta}\right\}$.

Given these assumptions, it can be easily seen that the function $b p$ is a homeomorphism.

DEFINITION 9: Let $p, q \in \mathbf{R}_{\Delta}^{2}$. We say that $p$ and $q$ are horizontally (resp. vertically) adjacent if $b p(p)$ and $b p(q)$ are adjacent and $\nu b p(p)=\nu b p(q)$ (resp. $h b p(p)=h b p(q))$. We say that $p$ is on the right of $q$ (resp. on the left) if $h b p(p)>h b p(q)$ (resp. $h b p(p)<h b p(q)$ ). We say that $p$ is above $q$ (resp. below) if $v b p(p)>v b p(q)$ (resp. $v b p(p)<v b p(q)$ ).

\section{CONTAINMENT RELATIONS}

\subsection{Containment between convex polygons}

In [CNT96] we characterized containment between convex polygons in $\mathbf{R}^{2}$ in terms of the containment between open sets of the topological space $\left(G_{\Delta}, \mathcal{T}_{\Delta}\right)$. We considered in [CNT96] only convex polygons such that $\overline{\sigma(\cdot)}$ is loop-connected.

We now briefly recall definitions and results from [CNT96]. Afterwards we give the treatment for nonconvex polygon and in the final section we generalize to polygons such that $\sigma(\cdot)$ is not loop-connected.

DEFINITION 10: Let $\mathcal{E}$ be the set of convex polygons in $\mathbf{R}^{2}$ whose vertices belongs to $G_{\Delta}$. Let $A \in \mathcal{E}$. We call skeleton of $A$ the set $\sigma(A)=\cup_{\mathbf{x}} U_{\mathbf{x}}$ for all $\mathrm{x} \in A \cap \mathcal{G}_{\Delta}$.

In figure 1 below examples of skeletons are shown. $\mathrm{A}$ circle indicates a point of $\mathcal{G}_{\Delta}$ that belongs to an open set, while a cross denotes points belonging to the boundary of an open set. Endpoints of lines are shown as grey circles.

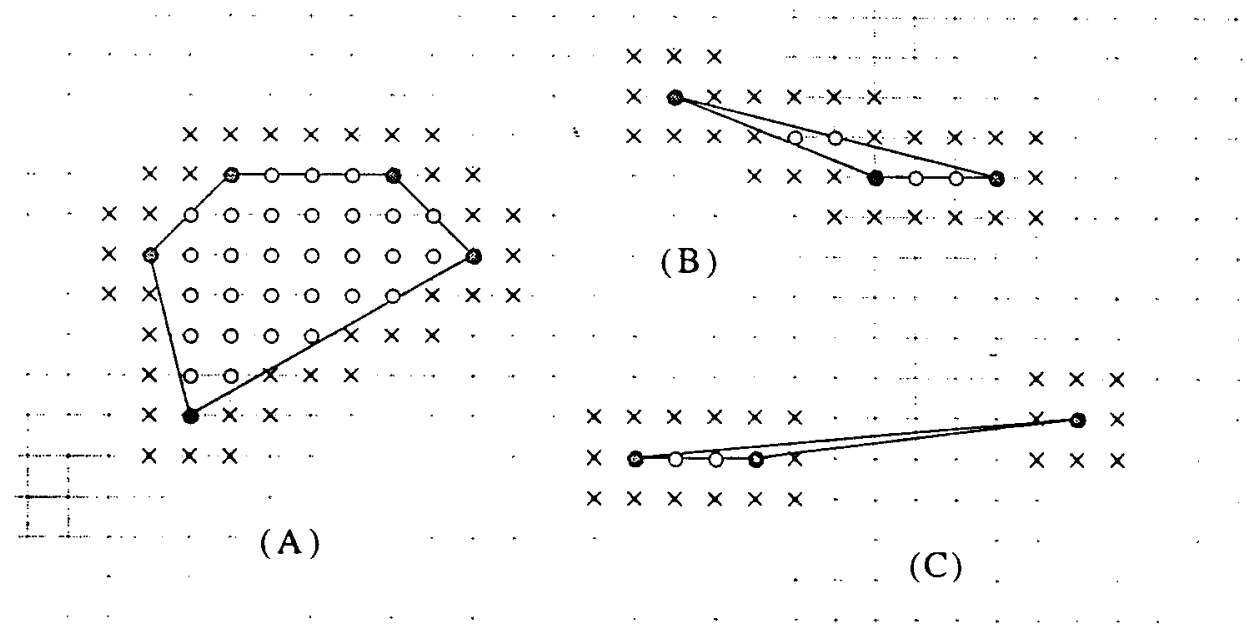

Figure 1: (A, B) - polygons whose skeletons have loop-connected closure:

(C) - a polygon whose skeleton has a closure that is not loop-connected.

The containment between two polygons in $\mathbf{R}^{2}$ is equivalent to the containment of their skeletons.

THEOREM 1 [CNT96]: Let $A, B \in \mathcal{E}$. Then $A \subseteq \mathcal{E}^{B}$ iff $\sigma(A) \subseteq G_{\Delta} \sigma(B)$.
Also, it is possible to define a mapping between $\mathbf{R}_{\Delta}^{2}$ and $Z^{2}$, namely $T: \mathbf{R}_{\Delta}^{2} \rightarrow \mathbf{Z}^{2}$, that satisfies the following conditions: 
1. $a=\mathrm{T}(p)$ and $b=\mathrm{T}(q)$ are horizontally (vertically) adjacent iff $p$ and $q$ are horizontally (vertically) adjacent;

2. $a$ is on the right (on the left) of $b$ iff $p$ is on the right (on the left) of $q$.

3. $a$ is above (below) $b$ iff $p$ is above (below) $q$.

Let $\mathcal{P}(\mathbf{S})$ denote the power set of set $\mathbf{S}$. Let us take $\mathbf{R}_{\Delta}^{2}$ and $\mathbf{Z}^{2}$ with their respective topologies, $\mathcal{T}_{\Delta}^{\prime}$ and $P\left(Z^{2}\right)$. Note that $T$ is open ${ }^{1}$. Moreover, $T$ is one-toone, since $\mathrm{T}(p) \neq \mathrm{T}(q)$ iff $p \neq q$.

THEOREM 2 [CNT96]: Let $A, B \in \mathcal{E}$. Then $A \subseteq \mathcal{E}^{B}$ iff $\mathrm{T}\left(b p^{-1}(\sigma(A))\right) \subseteq \mathrm{z}^{2 \mathrm{~T}}\left(b p^{-1}(\sigma(B))\right)$.

The above theorem then allows us to reduce testing of containment between convex polygons of $\mathbf{R}^{2}$ to the testing of set containment between their correspondent sets of points of $\mathbf{Z}^{2}$.

\subsection{Relations between line segments}

We now study relations between line segments with a twofold aim. First to be able to generalize to nonconvex polygons results obtained in the previous section, and second to prepare the way for the characterization of the intersection relation between arbitrary polygons. To obtain this we adopt a formal framework that is different from the one we have used in [CNT96].

Let $\mathcal{L}$ be the set of line segments of $\mathbf{R}^{2}$ whose endpoints belong to $\mathcal{G}_{\Delta}$. We denote with $l_{\infty}$ the infinite line corresponding to a line segment $l \in \mathcal{L}$. We denote with $l_{\infty}^{+}$and $l_{\infty}^{-}$respectively the right and left (according to some arbitrarily chosen direction for $l_{\infty}$ ) open halfplane of $\mathbf{R}^{2}$ defined by $l_{\infty}$. In this sub-section it is always $l, l^{\prime} \in \mathcal{L}$.

DEFINITION 11: The set $\mathcal{N}\left(l_{\infty}\right)=\left\{\mathbf{x} \in \mathcal{G}_{\Delta} \mid \exists \mathbf{y} \in \overline{U_{\mathbf{x}}}\right.$ : $\left.\left(\mathrm{y} \in l_{\infty}^{+} \wedge \mathrm{x} \in l_{\infty}^{-}\right) \vee\left(\mathrm{y} \in l_{\infty}^{-} \wedge \mathrm{x} \in l_{\infty}^{+}\right)\right\}$is called the set of the near points of $l_{\infty}$.

DEFINITION 12: Let $(a, b)$ and $(c, d)$ be the endpoints of $l$. The set $\mathcal{F}(l)=\left\{(x, y) \in \mathcal{N}\left(l_{\infty}\right)\right\} \min (a, c) \leq x \leq$ $\max (a, c) \wedge \min (b, d) \leq y \leq \max (b, d)\}$ is called the set of convexification points of $l$.

Note that if line segment $l$ is horizontal or vertical then the set $\mathcal{F}(l)$ is empty.

DEFINITION 13: We call skeleton of $l$ the set $\sigma(l)=\cup_{\mathbf{x}} U_{\mathbf{x}}$ for all $\mathbf{x} \in \ln \mathcal{G}_{\mathbf{j}}$. The set $\mathcal{H}(l)=\overline{\sigma(l)} \mathcal{F}(l)$ is called the envelope of $l$. The subset of $\mathcal{I}(l)$ that is

1 A map from a topological space $X$ to another topological space $Y$ is open iff the image of each open set of is an open set. contained in $l_{\infty}^{+}$(resp. $l_{\infty}^{-}$is called the right (resp. left) envelope of $l$ and is denoted $\mathcal{J}^{+}(l)$ (resp. $\mathcal{T}(l)$ ).

Note that the envelope of a segment line is a closed set and is always loop-connected.

We now characterize the intersection relation between line segments in terms of relations between their envelopes.

FACT 1: If $\mathcal{H}(l) \cap \mathcal{H}\left(l^{\prime}\right)=\varnothing$ then $l \cap l^{\prime}=\varnothing$.

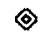

When the intersection between envelopes is not empty additional conditions are necessary since in this case line segments may have no intersection at all. If the intersection between envelopes contains both a point $x$ of $G_{\Delta}$ and the closure of $U_{\mathbf{x}}$ then the two line segments intersect at $x$. But this sufficient condition is not necessary since two line segments may intersect in a point $y$ not belonging to $G_{\Delta}$. To characterize these two different cases of intersection between line segments we introduce the following notation.

When the intersection between envelopes is not empty additional conditions are necessary since in this case line segments may have no intersection at all. If the intersection between envelopcs contains both a point $x$ of $\mathcal{G}_{\Delta}$ and the closure of $U_{\mathbf{x}}$ then the two line segments intersect at $x$. But this suivicient condition is not necessary since two line sec nents may intersect in a point $y$ not belonging to $\mathcal{G}_{\Delta}$. To characterize these two different cases of intersection between line segments we introduce the following notation.

We let $X\left(l, l^{\prime}\right)=\left\{\mathbf{x} \in \mathcal{G}_{\Delta} \mid \overline{U_{\mathbf{x}}} \subseteq \mathcal{J}(l) \cap \mathcal{J}\left(l^{\prime}\right)\right\}$. Informally speaking $\chi\left(l, l^{\prime}\right)$ is the set of grid points whose closed pointed neighbourhood belongs to the envelope of both segment lines.

We now introduce the notation $\operatorname{int}\left(l, l^{\prime}\right)$ which is true if and only if the following predicate is true:

$\left(\exists \mathbf{x} \in \sigma(l), \mathbf{y} \in \sigma(l) \mid \mathbf{x} \in \mathcal{I}^{-}\left(l^{\prime}\right) \wedge \mathbf{y} \in \mathcal{I}^{+}\left(l^{\prime}\right)\right) \wedge$ $\left(\exists \mathbf{w} \in \sigma(l), \mathbf{z} \in \sigma\left(l^{\prime}\right) \mid \mathbf{w} \in \mathcal{J}^{-}(l) \wedge \mathbf{z} \in \mathcal{J}^{+}(l)\right)$.

Informally speaking the boolean predicate int $\left(l, l^{\prime}\right)$ tells, when true, if both the two segment lines have at least one of their skeleton points in each the open halfplanes defined by the other segment line.

In figure 2 various possibilities for intersection between envelopes are shown: note that in case (A) $\operatorname{int}\left(l, l^{\prime}\right)$ is true while in case (B) and (C) int $\left(l, l^{\prime}\right)$ is false. Points belonging to $\mathcal{N}\left(l_{\infty}\right)$ are shown as small full circles, those belonging also to $\mathcal{F}(l)$ are within a square. 


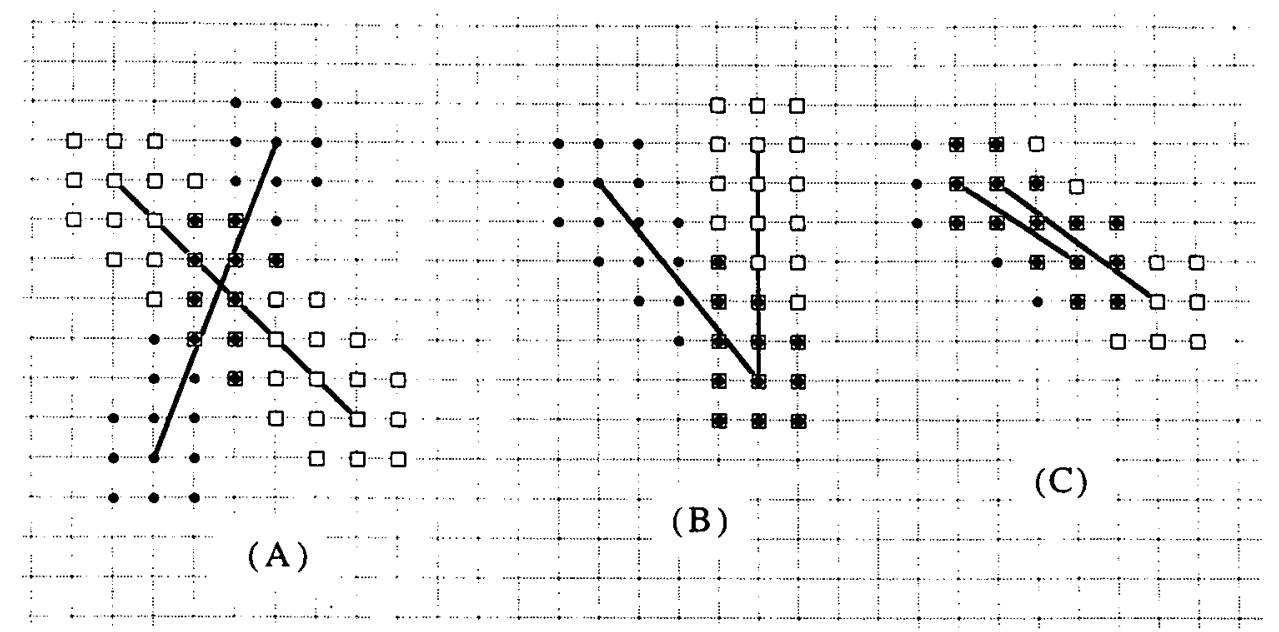

Figure 2: (A) - envelopes of two intersecting lines whose intersection point does not belong to $G_{\Delta}$; (B) - envelopes of two intersecting lines whose intesection point belongs to $\mathcal{G}_{\Delta} ;(\mathrm{C})$ - envelopes of two non intersecting lines.

Note also that the following two implications hold .

FACT 2: If $\mathcal{H}(l) \cap \mathcal{H}\left(l^{\prime}\right)=\varnothing$ then $\neg \operatorname{int}\left(l, l^{\prime}\right)$.

()

FACT 3: If $\operatorname{int}\left(l, l^{\prime}\right)$ then $\mathcal{I}(l) \cap \mathcal{X}\left(l^{\prime}\right) \neq \varnothing$.

The following theorem gives the desired characterization with reference to the case of a not empty intersection:

THEOREM 3: It is $l \cap l^{\prime} \neq \varnothing$ if and only if at least one of the following condition holds:

(C1) $\left.X\left(l, l^{\prime}\right)\right) \neq \varnothing$

(C2) $\quad \operatorname{int}\left(l, l^{\prime}\right)$ is true.

PROOF:

(IF) If $l \cap l^{\prime} \neq \varnothing$ then an $\mathrm{x}$ such that $\mathrm{x} \in \ln l^{\prime}$ exists. Two cases are possible: $\mathbf{x} \in \mathcal{G}_{\Delta}$ or $\mathbf{x \notin} \mathcal{G}_{\Delta}$. In the former case we have $X\left(l, l^{\prime}\right) \neq \varnothing$. In the latter case int $\left(l, l^{\prime}\right)$ must be true, otherwise its falsity would imply that at least one segment line has both its endpoints in exactly one of the open halfplanes defined by the other segment line, a contradiction.

(ONLY IF) The implication $\left.\chi\left(l, l^{\prime}\right)\right) \neq \varnothing \Rightarrow l \cap l^{\prime} \neq \varnothing$ follows by the definition of $X$, the remaining one follows by the definition of int $\left(l, l^{\prime}\right)$.

(-)

\subsection{Containment between arbitrary polygons}

In this sub-section we extend the results of subsection 3.1 by considering also relations between arbitrary (i.e.. not necessarily convex) polygons. For the sake of simplicity, we again first consider only convex polygons such that $\sigma(\cdot)$ is loop-connected, and we shall later remove this restriction.

To investigate containment relations between arbitrary polygons it is necessary to use the characterization of relations between line segments defined in the previous sub-section. In fact, it is not possible, working only with skeletons to determine containment. This is due to the fact that a convex polygon is uniquely identified by its skeleton while this is not true for concave polygons. Even if the number of sides of a concave polygon is known this is not sufficient to uniquely identify the polygon from its skeleton (see figure 3 ).

Let $\mathcal{E}^{\prime}$ be the set of all polygons in $\mathbf{R}^{2}$ whose vertices belongs to $G_{\Delta}$.

In this sub-section it is always $A, B \in \mathcal{E}^{\prime}, l \in \mathcal{L}, l^{\prime}$ a side of $A$, and $l^{\prime \prime}$ a side of $B$.

LEMMA 2: If $\operatorname{int}\left(l, l^{\prime}\right)$ then $l \Phi_{\mathcal{E}}, A$.

PROOF: If $\operatorname{int}\left(l, l^{\prime}\right)$ is true then $l \cap l^{\prime} \neq \varnothing$. Then we have two cases: either $l$ has at least one endpoint outside $A$ or $l$ has both the endpoints inside $A$. In the first case the thesis is trivially true. In the second case, since $l^{\prime}$ has to intersect some side of $A$, the only possibility is that $l$ intersects an even number of sides of $A$. It easy to check that this may happen only when $A$ is concave and $l$ is not completely contained in $A$ 


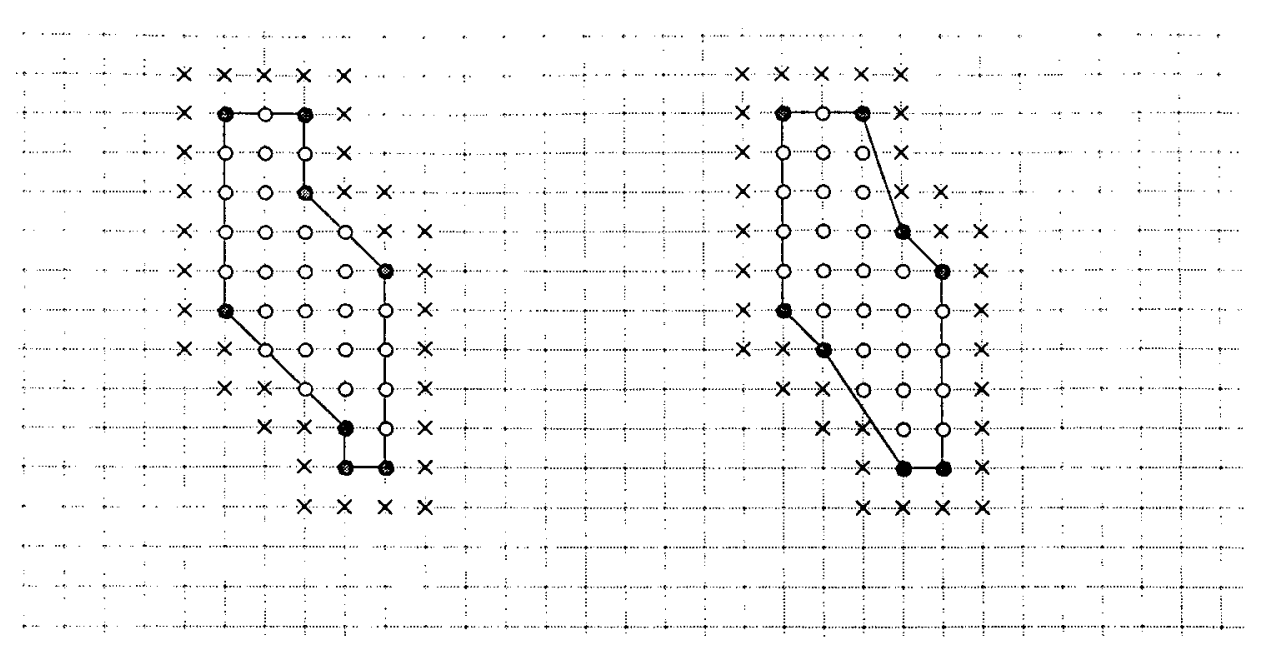

Figure 3: two different concave polygons with the same number of sides and the same skeleton.

COROLLARY 2: If for some $l^{\prime}, l^{\prime \prime}$ it is $\operatorname{int}\left(l, l^{\prime}\right)$ then $A \Phi_{\mathcal{E}}, B$.

FACT 5: If $A \subseteq \mathcal{E}^{\prime} B$ then $\sigma(A) \subseteq G_{\Delta} \sigma(B)$.

To have a containment between two arbitrary polygons, beyond the containment between their skeletons, we have to check that their sides are in a suitable relation. From an intuitive point of view, the condition is that if envelopes of their sides intersect, then their intersections have only to be in points of the grid that are endpoints for some side or to be coincident with some side or some part of it (see figure 4).
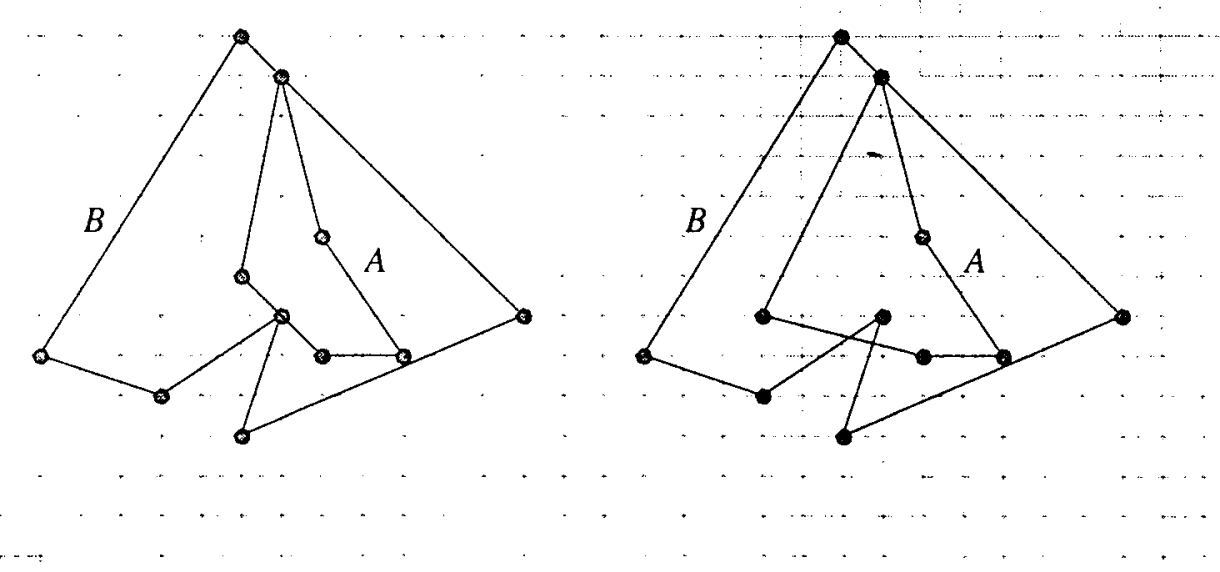

Figure 4: in both cases $\sigma(A) \subseteq \sigma(B)$, but in the left example $A$ is contained in $B$ while in the right one $A$ is not contained in $B$.

THEOREM 4: It is $A \subseteq \mathcal{T}^{\prime} B$ if and only if both the following conditions hold:

(C1) $\sigma(A) \subseteq G_{\Delta} \sigma(B)$

(C2) $\forall l^{\prime}, l^{\prime \prime}$, it is $\rightarrow \operatorname{int}\left(l^{\prime}, l^{\prime \prime}\right)$.

\section{PROOF:}

(IF): if both conditions are true then it follows, by lemma 2, that $A$ is contained in $B$.
(ONLY IF): if $A \subseteq \mathcal{E}^{, B}$ holds, then each $l^{\prime}$ side of $A$ is contained in $B$. Then, by lemma 2 , we have $\neg \operatorname{int}\left(l^{\prime}, l^{\prime \prime}\right)$. Moreover, $\mathrm{C} 1$ must be true: otherwise an $\mathbf{x} \in \mathcal{G}_{\Delta}$ exists such that $\mathbf{x} \in \sigma(B)$ and $\mathbf{x} \notin \sigma(A)$, a contradiction.

In a way analogous to the case of containment between convex polygons, i.e., by means of maps $\mathrm{T}$ and $b p$, we can reduce testing of containment between arbitrary polygons of $\mathbf{R}^{2}$ to the testing of set containment between their correspondents in $\mathbf{Z}^{2}$. 


\section{INTERSECTION RELATIONS}

We now study intersection relations between polygons of $\mathcal{E}^{\prime}$ using results derived in the previous section and building on the characterization of intersection between line segments.

In this section it is always $A, B \in \mathcal{E}^{\prime}$. Let $\Psi$ be denote a suitable degenerate open set of $\mathcal{T}_{\Delta}$. Once again we consider, for the time being, only convex polygons such that $\sigma(\cdot)$ is loop-connected.

PROPOSITION 1: If $A \cap B=\varnothing$ then $\sigma(A) \cap \sigma(B)=\varnothing \vee$ $\sigma(A) \cap \sigma(B)=\Psi$.

PROOF: If $A \cap B=\varnothing$ there is no point of $A \cap \mathcal{G}_{\Delta}$ that is belonging to $B \cap G_{\Delta}$ and viceversa. By definition of $\sigma$ we have the thesis.
For the viceversa we have to distinguish between the two cases of the intersection of the skeletons being the empty set or a degenerate open set. The first is simpler.

PROPOSITION 2: If $\sigma(A) \cap \sigma(B)=\varnothing$ then $A \cap B=\varnothing$.

PROOF: By hypothesis we have that $A \cap B \cap G_{\Delta}=\varnothing$. Let us then consider an $\mathbf{x} \notin \mathcal{G}_{\Delta}$. Assume, by absurd, that $\mathbf{x} \in A \cap B$. Then it has to exist a $\Psi$ such that be $\sigma(A) \cap \sigma(B) \supseteq \Psi$, a contradiction.

DEFINITION 14: Let $A, B \in \mathcal{E}$. We say $A$ and $B$ are near if $\sigma(A) \cap \sigma(B)$ is a degenerate open set.

When $A$ and $B$ are near it may happen that they intersect (e.g., when they are thin). See figure 5, where the degenerate open set is shown with bolder symbols.

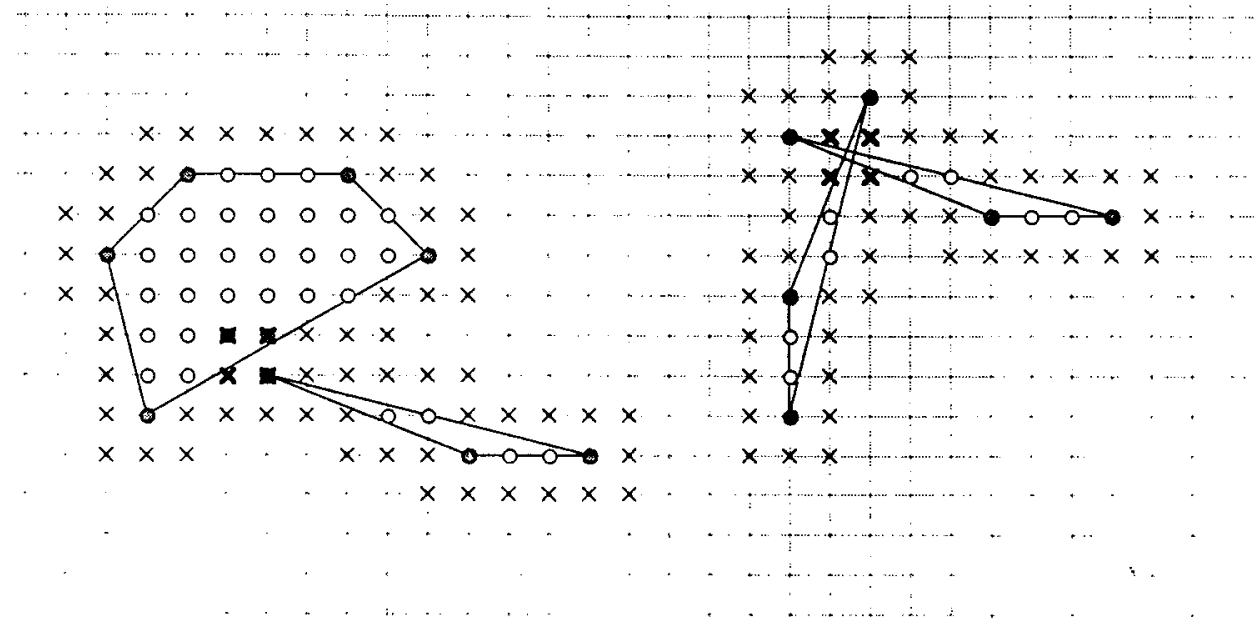

Figure 5: (left) two near polygons which are not intersecting; (right) two near polygons which are intersecting.

In the case of near polygons, if we want to distinguish disjointness from intersection, we therefore have to analyze the intersection relation among their boundaries. This is necessary only when polygons are "thin", since in this case their skeleton is not a complete representation of them.

DEFINITION 15: We say $A$ is fully represented by its skeleton if $\sigma(A)$ does not contain a degenerate open set.

For fully represented polygons, the characterization of intersection is provided by the following theorem, whose proof is straightforward.

THEOREM 5: Let $A$ be a polygon fully represented by its skeleton. It is $A \cap B \neq \varnothing$ if and only if an $\mathbf{x} \in \mathcal{G}_{\Delta}$ exists such that $\mathbf{x} \in \sigma(A) \cap \sigma(B)$.
Let $A$ and $B$ be two polygons such that at least one of them is not fully represented. Clearly, if $A$ and $B$ are intersecting in a point of $\mathcal{G}_{\Delta}$ then the intersection between their skeletons is not empty. The viceversa is not necessarily true: in fact, if the intersection between their skeletons contains only a degenerate set, we cannot conclude that $A$ and $B$ are disjoint. The following theorem, whose proof is straightforward, provides a characterization of intersection between arbitrary polygons (fully represented or not) belonging to $\mathcal{E}^{\prime}$.

THEOREM 6: Let $l^{\prime}$ be a side of $A$ and $l^{\prime \prime}$ be a side of $B$. It is $A \cap B \neq \varnothing$ if and only at least one of the following conditions are true:

$$
\exists \mathbf{x} \in \mathcal{G}_{\Delta} \mid \mathbf{x} \in \sigma(A) \cap \sigma(B)
$$




\section{GENERALIZATION OF THE MODEL}

We now discuss how to remove the restriction of loopconnectivity. If $A \in \mathcal{E}^{\prime}$ has a skeleton whose closure is not loop-connected, than we introduce a generalized skeleton, which includes $\sigma(A)$ and is loop-connected.

DEFINITION 16: The generalized skeleton of $A$, denoted $\sigma^{\prime}(A)$, is the largest open set of $T_{\Delta}$ that is contained in $\overline{\sigma(A)} \cup\left\{\cup_{l, l^{\prime}} \mathcal{H}(l) \cap \mathcal{H}\left(l^{\prime}\right)\right.$ for $l, l^{\prime}$ sides of $\left.A\right\}$.

Note that if the skeleton of a polygon is already loopconnected then the generalized skeleton coincides with the skeletons itself.

Let $A$ and $B$ be two polygons represented by means of generalized skeletons. In this case also, if the intersection between their generalized skeletons is empty, we cannot conclude that $A$ and $B$ are disjoint. It easy to prove that the interection relation is completely characterized by theorem 6 .

\section{Conclusions}

In this paper we have defined and characterized a topology preserving mapping from extended spatial objects to a discrete space. This allow us to correctly answer queries about topological relations of containment and intersection by manipulating the discrete representations of spatial objects: we have therefore extended to polygons the approach of Greene and Yao [GY86].

We are thus able to identify each of the three basic relative positions of spatial objects (namely: containment, intersection, and disjointness), which are fundamental for any efficient organization of spatial data.

We now sketch some of the most interesting directions for future research we are currently investigating. First of all, the study of computability and computional complexity issues relative to our model. Secondly, a formal characterization of relations among spatial objects whose endpoints are not bound to be points of $G_{\Delta}$ and among spatial objects which are not simply connected. Thirdly, a study of how our model characterizes other spatial relations and, more in general, supports spatial reasoning. Finally, an indepth analysis of relationships between our model and the formal models for topological relations introduced in the continuous space.

\section{REFERENCES}

[CDO93] E.Clementini, P.DiFelice, and P.vanOosterom. A small set of formal topological relationships suitable for end-user interaction. 3rd Int. Symp. on Large Spatial Databases (SSD'93), Singapore, 1993. Lecture Notes in Computer Science 692:277-295, Advances in Spatial Databases, edited by D.Abel and B.Ooi, Springer Verlag.

[CNT96] F.Coppa, E.Nardelli, M.Talamo, Discrete representation of spatial relations, I1th International Symposium on Computer and Information Sciences (ISCIS-XI), Antalya, Turkey, November 1996.

[EFJ89] M.J.Egenhofer, A.U.Frank, and A.P.Jackson. A topological data model for spatial databases. Ist Int. Symp. on Large Spatial Databases (SSD'89), Santa Barbara, CA, 1989. Lecture Notes in Computer Science 409:271-286, Design and Implementation of Large Spatial Databases, edited by A.Buchmann, O.Günther, T.R.Smith, and Y.-F.Wang, Springer Verlag.

[EF91] M.J.Egenhofer and R.D.Franzosa. Point-set topological spatial relations. Int. J. of Geographical Information Systems, 5(2):161-174, 1991.

[ES93] M.J.Egenhofer and J.Sharma. Topological relations between regions in $\mathbf{R}^{2}$ and $\mathbf{Z}^{2}$. 3rd Int. Symp. on Large Spatial Databases (SSD'93), Singapore, 1993. Lecture Notes in Computer Science 692:316-336, Advances in Spatial Databases, edited by D.Abel and B.Ooi, Springer Verlag.

[GY86] D.H.Greene and F.F.Yao. Finite-resolution computational geometry. 27th IEEE Symp. on Foundations of Computer Science (FOCS'86), Toronto, Canada, 143-152, 1986.

[GS93] R.H.Güting and M.Schneider. Realms: a foundation for spatial data types in database systems. 3rd Int. Symp. on Large Spatial Databases (SSD'93), Singapore, 1993. Lecture Notes in Computer Science 692:14-35, Advances in Spatial Databases, edited by D.Abel and B.Ooi, Springer Verlag.

[GSS89] L.Guibas, D.Salesin, and J.Stolfi. Epsilon Geometry: building robust algorithms from imprecise computations. 5th ACM Symp. on Computational Geometry, Saarbrucken, Germany, 208-217, 1989.

[GSS93] L.Guibas, D.Salesin, and J.Stolfi. Computing strongly convex approximate hulls with inaccurate primitives. Algorithmica, 9:534-560, 1993.

[Her91] J.R.Herring. The mathematical modeling of spatial and non-spatial information in Geographic Information Systems. Cognitive and linguistic aspects of geographic space, 313-350, edited by D.Mark and A.Frank. Kluwer Academic Publisher, Dordrecht, 1991.

[KEG] W.Kainz, M.J.Egenhofer, and I.Greasley. Modeling spatial relations and operations with partially ordered sets. Int. J. of Geographical Information Systems, to appear.

[Wor92] M.F.Worboys. A generic model for planar geographical objects. Int. J. of Geographical Information Sysicms, 6(5):353-372, 1992. 